\title{
ANGIOMIXOMA AGRESIVO DE VAGINA: REPORTE DE CASO Y REVISIÓN DE LA LITERATURA
}

\section{Aggressive angiomyxoma of the vagina: Case report and review of the literature}

\author{
Diana Meliza Hidalgo-Zambrano, M. D. ${ }^{1}$; Daniel Felipe Reyes-Vega, M. D. ${ }^{2}$; \\ Luis Guillermo Cano-Arias, M.D. ${ }^{3}$ \\ Recibido: 17 de abril de 2020/Aceptado: 17 de noviembre de 2020
}

\section{RESUMEN}

Objetivos: presentar el caso de una paciente con diagnóstico de angiomixoma agresivo de vagina, y hacer una revisión de la literatura del diagnóstico, tratamiento y pronóstico.

Materiales y métodos: mujer de 46 años de edad, quien consulta por disuria y sensación de masa en vagina. Tras la exploración física y los estudios imagenológicos se observa tumoración en vagina con extensión hacia cavidad pélvica, la cual es extraída por cirugía vaginal. La patología quirúrgica mostró angiomixoma agresivo de vagina. La lesión presentó recurrencia al tercer mes posoperatorio requiriendo una nueva cirugía por vía retrorrectal. Se realizó una búsqueda en las bases de datos Medline vía PubMed, LILACS, SciELO y Google Scholar, con los términos: "Angiomixoma", "Agresivo" y "Vagina",

* Correspondencia: Daniel Felipe Reyes Vega, Carrera 33 No. 18A-08, Barrio El Vergel, Neiva (Colombia). Teléfono: 3174246744 , danfereve@hotmail.com

1. Residente de Ginecología y Obstetricia, Universidad Surcolombiana, Neiva (Colombia).

2. Médico general, Hospital Universitario Hernando Moncaleano Perdomo, Neiva (Colombia).

3. Docente del Departamento de Ginecología, Universidad Surcolombiana, Unidad de Ginecología Oncológica, Hospital Universitario Hernando Moncaleano Perdomo, Neiva (Colombia). incluyendo artículos de revisión, reportes y series de caso en inglés y español publicados desde 1995. Se extrajo información sobre el diagnóstico referente a síntomas, signos, pruebas de inmunohistoquímica e imágenes utilizadas, el tipo de tratamiento -quirúrgico o de otro tipo- y el pronóstico. Se hace descripción narrativa de los hallazgos

Resultados: se identificaron 23 títulos, de los cuales 14 reportes de caso, 2 series de casos clínicos y un artículo de revisión cumplieron los criterios de inclusión. El $65 \%$ de las pacientes tenía entre 30 y 50 años. El diagnóstico se hizo con estudios de inmunohistoquímica en 8 de los casos y se utilizaron imágenes diagnósticas en 12 casos; la tomografía mostró mejor delimitación de las lesiones. El tratamiento quirúrgico se aplicó en todos los reportes y se complementó con terapia hormonal en 4 casos. Se hizo seguimiento a 14 de los 17 casos reportados. Conclusión: el angiomixoma agresivo de vagina es una entidad poco frecuente. Es necesario evaluar la utilidad de las diferentes pruebas de inmunohistoquímica en los casos identificados como angiomixoma agresivo por histopatología. Se requiere evaluación del tratamiento hormonal como coadyuvante de la cirugía. El pronóstico es bueno. 
Palabras clave: angiomixoma; vagina; tratamiento; pronóstico.

\section{ABSTRACT}

Objectives: To present the case of a patient diagnosed with aggressive angiomyxoma of the vagina and to conduct a review of the diagnosis, treatment and prognosis of this disease condition.

Materials and Methods: A 46-year old female patient complaining of dysuria and vaginal mass sensation. Physical exploration and imaging studies revealed a tumor extending into the pelvic cavity. The mass was resected through a vaginal approach and pathology of the surgical specimen showed a aggressive angiomyxoma of the vagina. New retrorectar surgery was performed three months after the initial resection because of recurrence. A search was conducted in the Medline via PubMed, Lilacs, Scielo and Google Scholar databases using the terms "Angiomyxoma," "Aggressive" and "Vagina." The search included review articles, case reports and case series published in English and Spanish since 1995. The information extracted included diagnosis, symptoms, signs, immunohistochemistry and imaging studies used, type of treatment - surgical or other - and prognosis. Findings are described in narrative form.

Results: Overall, 23 titles were identified, of which 14 case reports, 2 clinical case series and 1 review article met the inclusion criteria. Sixty-five per cent of the patients were between 30 and 50 years of age. Diagnosis was made by immunohistochemistry in 8 cases, and diagnostic imaging was used in 12 cases. Computed tomography showed sharper contours of the lesions. Surgical treatment was applied in all reports, supplemented by hormonal therapy in 4 cases. There was follow-up in 14 of the 17 cases reported.

Conclusion: Aggressive angiomyxoma of the vagina is rare. Assessment of the role of various immunohistochemical tests is needed in cases identified as aggressive angiomyxoma on histopathology.
Evaluation of hormonal treatment as an adjunct to surgery is required. Prognosis is good.

Key words: angiomyxoma; vagina; treatment; prognosis.

\section{INTRODUCCIÓN}

El angiomixoma agresivo (AA) es un tumor de origen mesenquimatoso mixoide, con marcada tendencia a la infiltración local y un alto porcentaje de recurrencias (1); es descrito por la Organización Mundial de la Salud (OMS) como una "neoplasia de tejidos blandos de diferenciación incierta” (2). Esta entidad aparece principalmente en tejidos blandos profundos (músculos, tendones, grasa, tejido fibroso, vasos y nervios) de la región pélvica, perineal e inguinal en mujeres adultas jóvenes (3), aunque también afecta en menor proporción a varones (relación mujeres/hombres 6,6/1) (1), en hombres se ubica generalmente en la región inguinoescrotal y en el periné (4). Fue descrito por primera vez en 1983 por Steeper y Rosai en nueve pacientes premenopáusicas (5), es una patología infrecuente, con menos de 350 casos reportados en la literatura (3). Se caracteriza por tener un crecimiento lento, localmente infiltrativo, a menudo recurrente, con pocos eventos metastásicos documentados hacia tejido pulmonar $(6,7)$.

El diagnóstico clínico es difícil debido a la presentación de síntomas inespecíficos y la amplia variabilidad en el tamaño tumoral, lo que dificulta también el diagnóstico diferencial (8). El diagnóstico definitivo se obtiene mediante histopatología; a la microscopia se observa una lesión mesenquimatosa hipocelular de células blandas fusiformes y estrelladas, esparcidas en un fondo de estroma mixoide suelto, compuesto por fibrillas de colágeno onduladas. No hay atipia citológica, ni características mitóticas atípicas o actividad mitótica discernible, y tampoco evidencia de necrosis de células tumorales coagulativas. Las células del estroma pueden mostrar inmunorreactividad a diferentes combinaciones de vimentina, desmina, actina de músculo 
liso, actina específica de músculo, CD 34 (9,10), y expresar positividad para receptores de estrógeno y progesterona (11). En cuanto a las imágenes diagnósticas, estas no son características. Jayadeban et al. (12), en 5 pacientes, reportan estudios de ultrasonido que suelen mostrar una masa hipoecoica de tejido blando o una imagen quística. La tomografía computarizada (TC) muestra una masa homogénea bien definida, hipodensa en relación con el músculo, o una masa sólida hipoatenuante con patrón interno arremolinado con contraste o también una lesión de apariencia predominantemente quística con componentes sólidos. Por último, estos autores indican que las imágenes de resonancia magnética nuclear $(\mathrm{RMN})$ son precisas para detectar la diseminación del tumor al piso pélvico o hacia las estructuras adyacentes, lo que permite una mejor planificación preoperatoria (12).

El tratamiento de elección consiste en la escisión quirúrgica de bordes amplios (2). Adicionalmente, ha sido documentada la administración de antagonistas de estrógenos y progestágenos cuando el tumor muestra positividad para receptores de estas hormonas (10); también se ha descrito el uso agonistas de la hormona liberadora de gonadotropinas (GnRH) para disminuir el tamaño del tumor (13). El pronóstico es bueno, a pesar del alto índice de recurrencias (14).

La información publicada sobre el AA de vagina es escasa, con poco más de 30 reportes de caso descritos en la literatura para 2012 (15). La baja incidencia del AA de vagina, su presentación clínica inespecífica y su tendencia a las recurrencias plantean un desafío diagnóstico y terapéutico para el ginecólogo. El objetivo de este estudio es describir el caso de una paciente con diagnóstico de AA de vagina y hacer una revisión de la literatura en cuanto al diagnóstico, tratamiento y pronóstico de esta entidad.

\section{PRESENTACIÓN DEL CASO}

Paciente de 46 años, quien acudió en marzo de 2018 al servicio de Ginecología del Hospital Universitario de Neiva, institución pública de alta complejidad, de referencia, ubicada en la región sur de país, que atiende pacientes del régimen subsidiado por el Estado y del contributivo de los trabajadores en el Sistema General de Seguridad Social de Colombia. Consultó por presencia de una masa sólida, no dolorosa, en pared vaginal izquierda, de tres años de evolución, que en los últimos siete meses había aumentado de tamaño, lo que generó disuria, dispareunia y sensación de masa. Al ingreso la frecuencia cardiaca era de 73 latidos por minuto (lpm), frecuencia respiratoria de 18 respiraciones por minuto (rpm), con una tensión de 113/68 milímetros de mercurio ( $\mathrm{mmHg}$ ), $58 \mathrm{~kg}$ de peso, se encontraba en buenas condiciones generales y no refería molestias adicionales. La paciente refería como antecedentes ginecológicos tres embarazos y tres partos, además de antecedente de histerectomía abdominal total y resección de un quiste ovárico derecho cinco años atrás. Al examen físico, el tacto vaginal reveló una masa que sobresalía en la pared lateral izquierda del tercio medio de la vagina, de forma irregular y consistencia cauchosa, no reductible, poco móvil, de aproximadamente $10 \times 12 \mathrm{~cm}$.

Con base en los hallazgos se consideró como primera impresión diagnóstica un tumor vaginal de carácter lipomatoso. Se solicitó una ecografía transvaginal que reportó la presencia de una imagen heterogénea, hipoecoica, de bordes mal definidos de $98 \times 63$ x $61 \mathrm{~mm}$, con una cápsula de $2 \mathrm{~mm}$ que se extendía desde tercio medio de la pared vaginal hacia el anexo izquierdo, con compromiso aparente del psoas. Ante la sospecha de un posible sarcoma del psoas, se realizó una RMN de pelvis contrastada, que reportó una lesión sólida, hiperintensa en las secuencias potenciadas en T2, con realce de manera homogénea y dimensiones de 11 x 11 x $8 \mathrm{~cm}$ con componente intra y extra-pélvico (figura 1), cuyas características insinuaban naturaleza mixoide. Dados los rasgos imagenológicos de la lesión, tamaño y localización, se definió la necesidad de manejo quirúrgico.

La paciente fue sometida a cirugía electiva en febrero de 2019, mediante abordaje transvaginal 

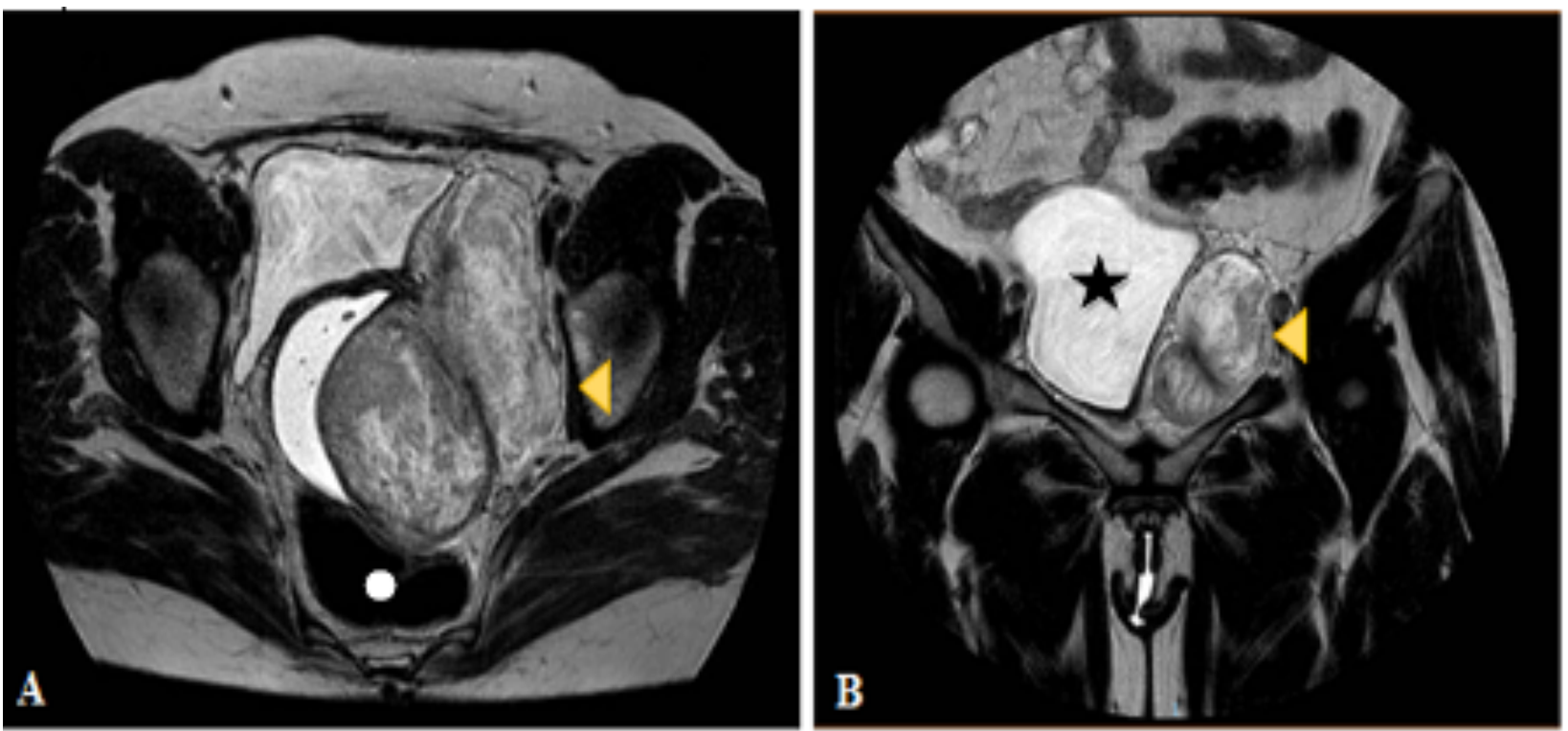

Figura 1. Resonancia Magnética de pelvis contrastada, (A) corte coronal, (B) corte axial. Se aprecia lesión hiperintensa heterogénea con contornos bien definidos de $11 \times 11 \times 8 \mathrm{~cm}$ (flecha naranja) que se extiende desde la grasa pélvica comprimiendo la pared lateral izquierda de la vejiga (estrella) y el recto inferior (círculo blanco). Técnica: RMN potenciada en T2 con eco espín rápido (4721/90, 1,5 Tesla)

fue identificada una masa de consistencia hialina, de $13 \times 12 \mathrm{~cm}$ de diámetro, poco vascularizada, que protruía por el fondo de saco lateral izquierdo del tercio superior de la vagina hacia cavidad pélvica. Se procedió a la resección amplia de la masa con toma de biopsia por congelación, que reportó un tumor estromal angiomixoide; adicionalmente, se enviaron muestras para análisis histopatológico. La paciente recibió manejo analgésico, antiemético y antibiótico durante dos días; presentó una adecuada recuperación, con alta hospitalaria en el tercer día posoperatorio. $\mathrm{Al}$ mes siguiente se realizó una consulta de control en la cual, al tacto vaginal, se encontró herida quirúrgica en buen estado sin dehiscencias o sangrado, ni presencia de masas.

El resultado final de patología reportó: lesión mesenquimal consistente con angiomixoma profundo agresivo; margen quirúrgico: en contacto con la lesión, necrosis: ausente, índice mitótico: $0 / 10$, diferenciación tumoral: 1, grado 3/8. La inmunohistoquímica reportó células fusiformes positivas para receptores de estrógenos y progesterona, con expresión heterogénea para "cluster" de diferenciación (CD) 34, desmina, actina de músculo liso y H-caldesmon, y negativa para CD31, factor de transcripción Sox10, CDK-4, con índice de proliferación celular de $2 \%$, medido por proteína KI67. Teniendo en cuenta los resultados de patología se solicitó una cita de control tres meses después.

Durante la revisión al tercer mes posoperatorio, la paciente se encontraba asintomática, con signos vitales dentro de la normalidad. Sin embargo, al tacto vaginal se identificó un área indurada nodular e irregular, en pared vaginal izquierda; una segunda RMN de pelvis reportó una lesión de aspecto mesenquimal, no infiltrativa, hiperintensa en las secuencias potenciadas en T2, de aproximadamente $8 \times 8 \times 8 \mathrm{~cm}$, que desplazaba la pared vaginal izquierda, el paquete vascular iliaco externo izquierdo, el recto inferior y la vejiga. Ante sospecha de recidiva tumoral se solicitó una ecografía endoscópica ano-rectal y se programó un control por junta oncológica tres meses después. $\mathrm{Al}$ sexto mes de la cirugía inicial se llevó a cabo un nuevo control por la junta oncológica de la institución, durante la revisión la paciente solo refirió sensación de masa en canal vaginal. El tacto vaginal fue similar 
a los realizados tres meses antes. El reporte de la ecografía endoscópica ano-rectal informó una lesión heterogénea de $6 \times 2,5 \mathrm{~cm}$, íntimamente adherida a la pared rectal distal con compromiso del tabique rectovaginal y del esfínter anal externo en la región lateral derecha. Se consideró paciente con recidiva tumoral confirmada, y se ordenó inicio de tratamiento con análogos de hormona liberadora de gonadotropina (GnRH) durante tres meses.

En marzo de 2020 se llevó a cabo una nueva intervención quirúrgica, se realizó un abordaje por vía retrorrectal, resecando una masa de consistencia hialina de $8 \times 6 \mathrm{~cm}$ de diámetro ubicada en la pared distal del recto, ampliando el margen quirúrgico de resección sin afectar el esfínter externo. Fue dada de alta 24 horas después, sin complicaciones. A los cuatro meses siguientes la paciente no había presentado nuevas recidivas, continuaba tratamiento con análogos de GnRH y a la espera de su próximo control por oncología con nueva RMN en cuatro meses.

Aspectos éticos. Se solicitó autorización a la paciente para la publicación de las imágenes y los datos clínicos contenidos en el reporte de caso, diligenciando el respectivo consentimiento informado. Durante el proceso se garantizó la confidencialidad de la información y el anonimato de la paciente, para proteger sus derechos de privacidad.

\section{MATERIALES Y MÉTODOS}

Con base en la pregunta: ¿cuál es el diagnóstico, tratamiento y pronóstico para el AA localizado en vagina?, se realizó una búsqueda bibliográfica en las bases de datos electrónicas de Medline vía PubMed, LILACS, SciELO y Google Scholar empleando los términos: "Angiomixoma", "Agresivo" y "Vagina". Se incluyeron artículos de revisión bibliográfica, reportes de caso y series de caso en inglés y español, desde enero de 1995 hasta julio de 2020, en mujeres de cualquier edad con AA con localización vaginal y con información del diagnóstico, tratamiento y pronóstico; se excluyeron artículos aún no publicados, estudios en los que no se dispuso del texto completo y estudios que reportaran AA en otra localización.
El proceso de selección se realizó en dos etapas, inicialmente se eligieron los artículos por título y resumen, y se obtuvo el texto completo de aquellos que cumplían los criterios de selección. Posteriormente, se revisó el contenido de los manuscritos para su inclusión definitiva. Las variables evaluadas fueron: diagnóstico en cuanto a síntomas, hallazgos en la inmunohistoquímica e imagenológicos, tratamiento quirúrgico y no quirúrgico, terapia adyuvante y pronóstico: tiempo de seguimiento, técnicas para el seguimiento, presencia de recurrencias, tratamiento de recurrencias, presencia de metástasis. Finalmente, se describieron los hallazgos.

\section{RESULTADOS}

Se identificaron inicialmente 23 artículos, de los que finalmente se incluyeron 17 publicaciones que cumplieron con los criterios de inclusión en el texto completo: 14 reportes de caso (15-28), dos series de casos clínicos $(29,30)$, con un único caso de localización vaginal descrito en cada uno, y una revisión de la literatura a partir de casos clínicos (31). En 3 estudios no se consiguió el texto completo (32-34).

Todos los artículos son publicaciones en inglés (15-28), excepto dos, que están escritos en español $(18,31)$. De los 17 casos reportados, dos se presentaron en pacientes entre los 20 y 29 años $(19,30)$, seis casos se presentaron en pacientes entre los 30 y 39 años (18,24,27-29,31), cinco casos en pacientes entre los 40 y $49(16,17,20,22,23)$ y cuatro casos en personas mayores de 50 años $(15,21,25,26)$.

Diagnóstico: en cuanto a los síntomas se identificó que seis de los casos reportaron masa dolorosa $(16,18,19,23,28,31)$, en cinco casos se presentaron alteraciones durante la micción $(17,19,20,21,25)$, en tres casos sensación de cuerpo extraño en vagina $(17,18,28)$, dispareunia en dos casos $(18,30)$ y un caso en el que se reportó dificultad para la deambulación (18). El tumor fue inicialmente diagnosticado como otra enfermedad del tracto genitourinario en todos los casos, previo a la confirmación diagnóstica por histopatología (tabla 1). 


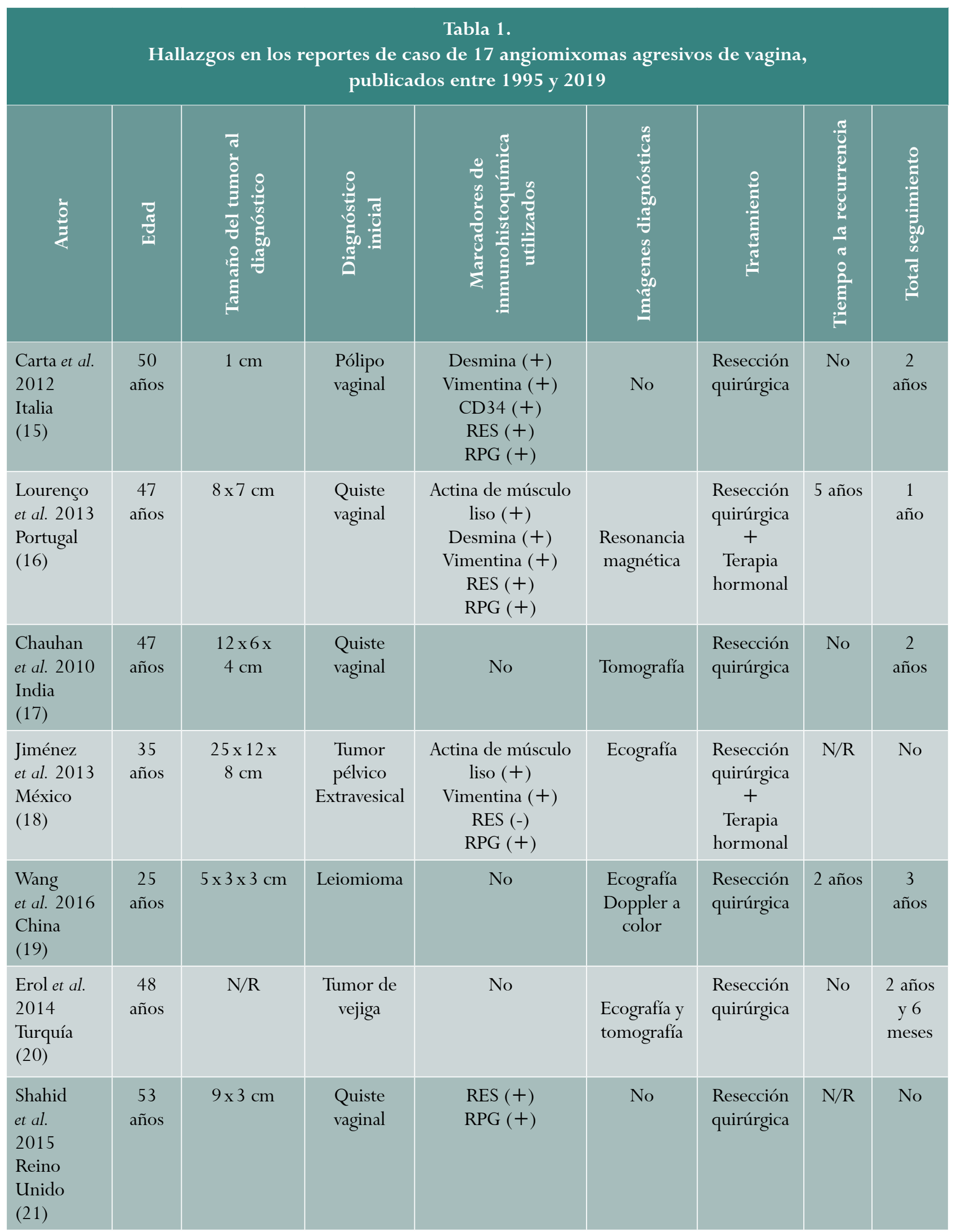




\begin{tabular}{|c|c|c|c|c|c|c|c|c|}
\hline$\frac{\ddot{0}}{2}$ & స్ & 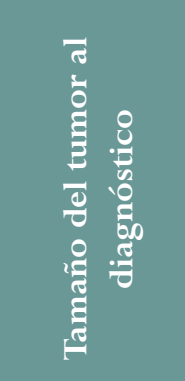 & 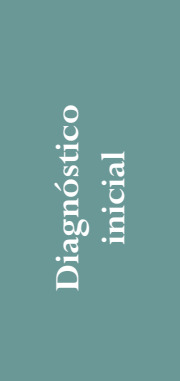 & 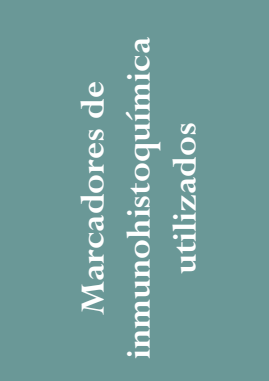 & 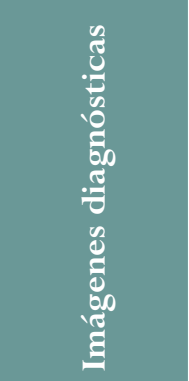 & 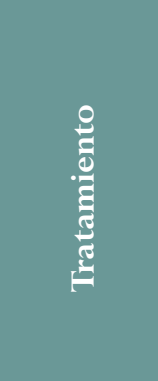 & 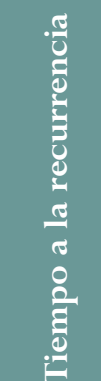 & 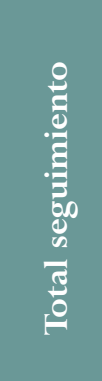 \\
\hline $\begin{array}{l}\text { Stumpf } \\
\text { et al. } \\
2016 \\
\text { Brasil } \\
(22)\end{array}$ & $\begin{array}{c}42 \\
\text { años }\end{array}$ & $\begin{array}{c}0,7 \times 0,6 \times \\
0,4 \mathrm{~cm}\end{array}$ & $\begin{array}{l}\text { Pólipo } \\
\text { vaginal }\end{array}$ & $\begin{array}{c}\text { Actina de músculo } \\
\text { liso }(-) \\
\text { Desmina }(+) \\
\text { RES }(+) \\
\text { RPG }(+) \\
\text { CDK4 }(+) \\
\text { CD34 }(+)\end{array}$ & $\begin{array}{c}\text { Resonancia } \\
\text { magnética }\end{array}$ & $\begin{array}{l}\text { Resección } \\
\text { quirúrgica }\end{array}$ & No & $\begin{array}{c}2 \\
\text { años }\end{array}$ \\
\hline $\begin{array}{l}\text { Seppings } \\
\text { et al. } 2017 \\
\text { Reino } \\
\text { Unido } \\
(23)\end{array}$ & $\begin{array}{c}46 \\
\text { años }\end{array}$ & $\begin{array}{c}9 \times 6 \\
\mathrm{~cm}\end{array}$ & $\begin{array}{l}\text { Quiste } \\
\text { vaginal }\end{array}$ & $\begin{array}{c}\text { Actina de músculo } \\
\text { liso }(+) \\
\text { Vimentina }(+) \\
\text { Desmina }(+) \\
\text { RES }(-) \\
\text { RPG }(+)\end{array}$ & $\begin{array}{l}\text { Ecografía y } \\
\text { resonancia } \\
\text { magnética }\end{array}$ & $\begin{array}{c}\text { Resección } \\
\text { quirúrgica } \\
+ \\
\text { Terapia } \\
\text { hormonal }\end{array}$ & No & $\begin{array}{c}3 \\
\text { meses }\end{array}$ \\
\hline $\begin{array}{l}\text { Cinel et al. } \\
2000 \\
\text { Turquía } \\
(24)\end{array}$ & $\begin{array}{c}38 \\
\text { años }\end{array}$ & $\begin{array}{c}9 \times 6 \times 2 \\
\mathrm{~cm}\end{array}$ & $\begin{array}{l}\text { Quiste } \\
\text { vaginal }\end{array}$ & No & No & $\begin{array}{l}\text { Resección } \\
\text { quirúrgica }\end{array}$ & No & $\begin{array}{c}3 \\
\text { años }\end{array}$ \\
\hline $\begin{array}{l}\text { Larouche } \\
\text { et al. } \\
2016 \\
\text { Canadá } \\
(25)\end{array}$ & $\begin{array}{c}66 \\
\text { años }\end{array}$ & $\begin{array}{c}12 \mathrm{x} 10 \mathrm{x} \\
4,5 \mathrm{~cm}\end{array}$ & $\begin{array}{c}\text { Hernia de } \\
\text { ovario y } \\
\text { ligamento } \\
\text { ancho }\end{array}$ & No & $\begin{array}{c}\text { Tomografía } \\
\text { y resonancia } \\
\text { magnética }\end{array}$ & $\begin{array}{l}\text { Resección } \\
\text { quirúrgica }\end{array}$ & No & $\begin{array}{c}3 \\
\text { meses }\end{array}$ \\
\hline $\begin{array}{l}\text { Amr et al. } \\
\text { Arabia } \\
\text { Saudita } \\
1995 \\
(26)\end{array}$ & $\begin{array}{c}50 \\
\text { años }\end{array}$ & $\begin{array}{c}11 \times 6,5 \times 3 \\
\mathrm{~cm}\end{array}$ & $\begin{array}{l}\text { Quiste } \\
\text { vaginal }\end{array}$ & No & $\begin{array}{l}\text { Ecografía y } \\
\text { tomografía }\end{array}$ & $\begin{array}{l}\text { Resección } \\
\text { quirúrgica }\end{array}$ & No & $\begin{array}{c}3 \\
\text { años }\end{array}$ \\
\hline $\begin{array}{l}\text { Gonzaga } \\
\text { et al. Brasil } \\
2005 \\
(27)\end{array}$ & $\begin{array}{c}32 \\
\text { años }\end{array}$ & $\mathrm{N} / \mathrm{R}$ & $\begin{array}{c}\text { Rabdomio- } \\
\text { sarcoma }\end{array}$ & CD $34(+)$ & Tomografía & $\begin{array}{l}\text { Resección } \\
\text { quirúrgica }\end{array}$ & 3 años & $\begin{array}{c}2 \\
\text { años }\end{array}$ \\
\hline $\begin{array}{l}\text { Manandhar } \\
\text { et al. Nepal } \\
2019 \\
(28)\end{array}$ & $\begin{array}{c}35 \\
\text { años }\end{array}$ & $0,5 \times 0,5 \mathrm{~cm}$ & $\begin{array}{l}\text { Cicatriz de } \\
\text { episiotomía }\end{array}$ & No & Ecografía & $\begin{array}{l}\text { Resección } \\
\text { quirúrgica }\end{array}$ & $\mathrm{N} / \mathrm{R}$ & No \\
\hline $\begin{array}{l}\text { Güngör } \\
\text { et al. } \\
\text { Turquía } \\
2004 \\
(29)\end{array}$ & $\begin{array}{c}38 \\
\text { años }\end{array}$ & $\begin{array}{c}10 \times 7 \times 3 \\
\mathrm{~cm}\end{array}$ & $\begin{array}{l}\text { Quiste } \\
\text { vaginal }\end{array}$ & No & No & $\begin{array}{l}\text { Resección } \\
\text { quirúrgica }\end{array}$ & 1 año & $\begin{array}{c}4 \\
\text { años }\end{array}$ \\
\hline
\end{tabular}




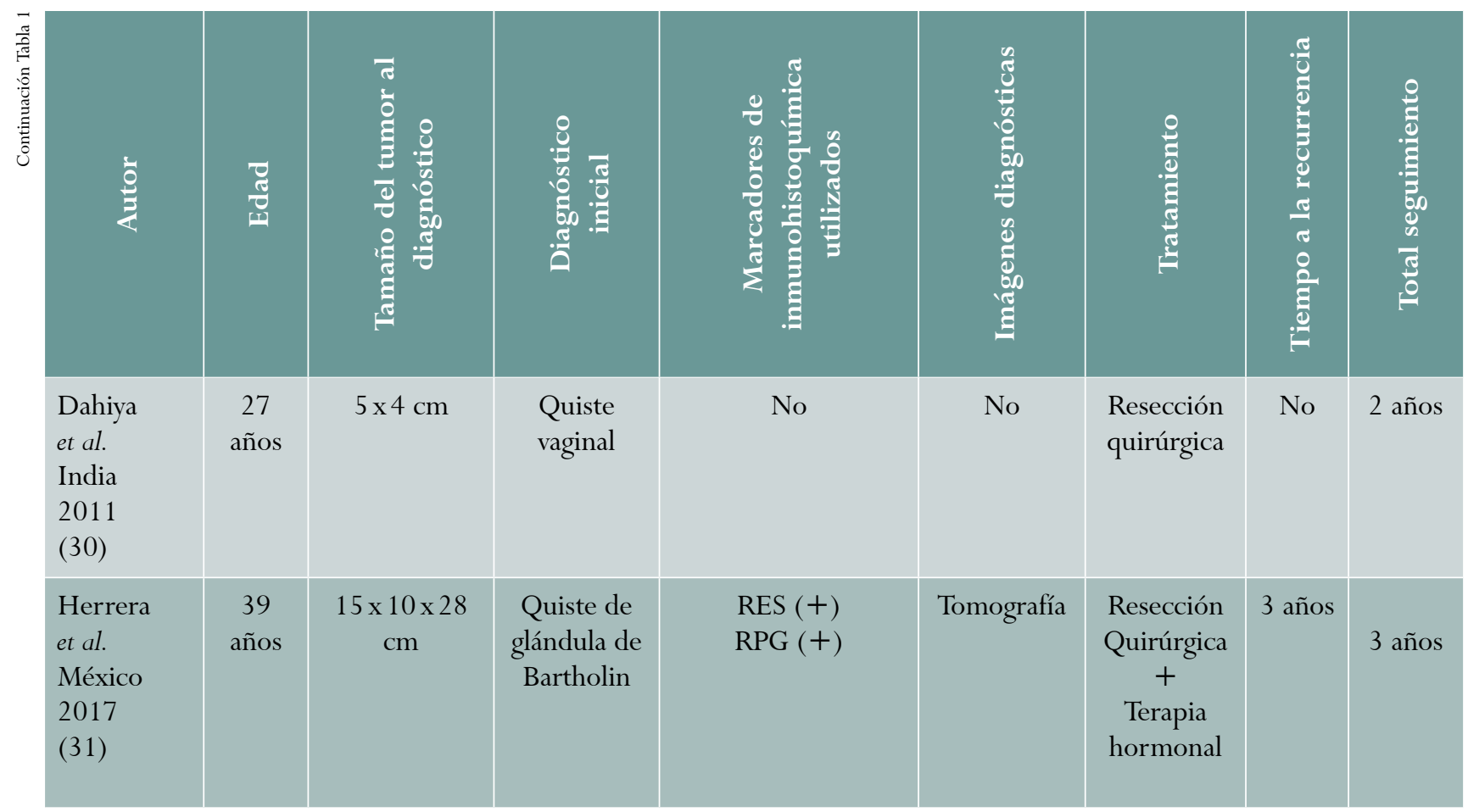

RES: receptores de estrógenos. RPG: receptores de progesterona. N/R: no registrado. CD: cúmulo de diferenciación.

Diagnóstico de histopatología: el diagnóstico se hizo con estudios de inmunohistoquímica en un total de ocho casos $(15,16,18,21-23,27,31)$. En los reportes se evaluaron diferentes marcadores asociados con la enfermedad, entre ellos hubo cuatro casos positivos para desmina $(15,16,22,23)$, cuatro casos positivos para vimentina $(15,16,18,23)$, tres positivos para actina de músculo liso $(16,18,23)$, siete casos positivos para receptores de progesterona $(15,16,18,21-23,31)$ y cinco positivos para receptores de estrógenos $(15,16,21,22,31)$; adicionalmente, el marcador CD 34 fue positivo en tres casos $(15,22,27)$. En nuestra paciente fueron positivos marcadores para receptores de estrógenos y progesterona, CD 34, desmina, actina de músculo liso y $\mathrm{H}$-caldesmon.

Diagnóstico mediante técnicas de imagen: en cuanto a estudios imagenológicos, se solicitó RNM en cuatro casos $(16,22,23,25)$ en los que se encontró un patrón común de lesión heterogénea, con estructura interna arremolinada y márgenes mal definidos, además de un aumento en la intensidad de la señal, con imágenes potenciadas en T2. En cinco eventos se realizó ultrasonido abdomino-pélvico $(19,20,23,26,28)$, que reportó lesiones hipocecoicas, mal delimitadas, de apariencia quística. En seis casos se ordenó tomografía axial computarizada (TAC) $(17,20,25-$ 27,31), las cuales reportaban una tumoración heterogénea, de escaso realce, hipodensa en relación con el músculo, altamente vascularizada, que por lo general producía desplazamiento de estructuras adyacentes y, finalmente, en el caso donde se realizó ecografía Doppler a color (19) se reportó un flujo sanguíneo arterial de baja resistencia en el entramado vascular. En cinco casos no se reportó uso de recursos imagenológicos $(15,21,24,29,30)$.

Estrategias terapéuticas: en todos los casos revisados los pacientes se sometieron a resección quirúrgica local, en cuatro casos se usó terapia hormonal junto con el tratamiento quirúrgico $(16,18,23,31)$. En dos casos $(16,27)$ fueron necesarias resecciones quirúrgicas mayores, incluyendo histerectomía, salpingooforectomía bilateral (16) y exanteración pélvica con linfadenectomía inguinal bilateral (27).

Pronóstico: nuestro análisis encontró 14 casos en los que se realizó seguimiento a las pacientes $(15,16,17,19,20,22-27,29-31)$ y tres en donde no se 
realizó o no fue reportado $(18,21,28)$. De los casos que hicieron seguimiento, cinco presentaron recidivas; cuatro aparecieron en los primeros tres años $(19,27,29,31)$ y una ocurrió cinco años después del tratamiento inicial (16). Adicionalmente, se observó un monitoreo durante al menos tres años en seis casos $(19,20,24,26,29,31)$, dos años en cinco casos $(17,15,22,27,30)$ y tres reportes con seguimientos de un año o menos hasta el momento de la publicación $(16,23,25)$. No se presentaron casos fatales o presencia de metástasis en ninguna de las pacientes.

\section{CONCLUSIONES}

El AA es una entidad bastante infrecuente, más aún en localización vaginal. Es necesario evaluar la utilidad de las diferentes pruebas de inmunohistoquímica en los casos identificados como AA por histopatología. Se requiere evaluación del tratamiento hormonal como coadyuvante de la cirugía.

\section{FINANCIACIÓN}

Este proyecto no contó con fuentes de financiación.

\section{REFERENCIAS}

1. Chan I, Hon E, Ngai SW, Ng TY, Wong L. Aggressive angiomyxoma in females: Is radical resection the only option? Acta Obstet Gynecol Scand. 2000;79:216-220. https://doi.org/10.1080/j.16000412.2000.079003216.x

2. Fletcher CDM, Unni KK, Mertens F., editors. World Health Organization Classification of Tumours. Pathology and Genetics of Tumours of Soft Tissue and Bone. Lyon: IARC Press; 2002.

3. Sutton BJ, Laudadio J. Aggressive angiomyxoma. Arch Pathol Lab Med. 2012;136(2):217-21. https://doi. org/10.5858/arpa.2011-0056-RS

4. Iezzoni JC, Fechner RE, Wong LS, Rosai J. Aggressive angiomyxoma in males. A report of four cases. Am J Clin Pathol. 1995;104(4):391-6. https://doi. org/10.1093/ajcp/104.4.391

5. Steeper TA, Rosai J. Aggressive angiomyxoma of the female pelvis and perineum. Report of nine cases of a distinctive type of gynecologic soft-tissue neoplasm.
Am. J. Surg. Pathol. 1983;7(5):463-75. https://doi. org/10.1097/00000478-198307000-00009

6. Blandamura S, Cruz J, Faure -Vergara L, MachadoPuerto I, Ninfo V. Aggressive angiomyxoma: A second case of metastasis with patient's death. Hum Pathol. 2003;34(10):1072-4. https://doi.org/10.1053/s00468177(03)00419-2

7. Siassi RM, Papadopoulos T, Matzel KE. Metastasizing aggressive angiomyxoma. N Engl J Med. 1999;341(23):1772. https://doi.org/10.1056/ nejm199912023412315

8. Hajjar R, Alharthi M, Richard C, Gougeon F, Loungnarath R. Pelvic aggressive angiomyxoma: Major challenges in diagnosis and treatment. $\mathrm{Cu}-$ reus. 2019;11(4):e4419. https://doi.org/10.7759/ cureus. 4419

9. Fetsch JF, Laskin WB, Lefkowitz M, Kindblom LG, Meis-Kindblom JM. Aggressive angiomyxoma: A clinicopathologic study of 29 female patients. Cancer. 1996;78(1):79-90. https://doi.org/10.1002/ (SICI) 1097-0142(19960701)78:1<79::AIDCNCR13>3.0.CO;2-4

10. Haldar K, Martinek IE, Kehoe S. Aggressive angiomyxoma: A case series and literature review. Eur J Surg Oncol. 2010;36(4):335-9. https://doi.org/10.1016/j. ejso.2009.11.006

11. Amezcua CA, Begley SJ, Mata N, Felix JC, Ballard CA. Aggressive angiomyxoma of the female genital tract: A clinicopathologic and immunohistochemical study of 12 cases. Int J Gynecol Cancer. 2005;15(1):140-5. https://doi.org/10.1111/j.1048-891x.2005.15015.x

12. Jeyadevan NN, Sohaib SAA, Thomas JM, Jeyarajah A, Shepherd JH, Fisher C. Imaging features of aggressive angiomyxoma. Clin Radiol. 2003;58(2):157-62. https://doi.org/10.1053/crad.2002.1127

13. McCluggage WG, Jamieson T, Dobbs SP, Grey A. Aggressive angiomyxoma of the vulva: Dramatic response to gonadotropin-releasing hormone agonist therapy. Gynecol Oncol. 2006;100(3):623-5. https://doi. org/10.1016/j.ygyno.2005.09.033

14. Bai HM, Yang JX, Huang HF, Cao DY, Chen J, Yang N, Lang JH, Shen K. Individualized managing strategies of aggressive angiomyxoma of female genital tract and 
pelvis. Eur J Surg Oncol. 2013;39(10):1101-8. https:// doi.org/10.1016/j.ejso.2013.06.013

15. Carta G, Parisse V, Accurti V, Sollima L, Di Stefano L, Alfonso DA, et al. Aggressive angiomyxoma of the vaginal wall at the initial stage: A case report. Europ J Gynaecol Oncol. 2012;33(6):669-71.

16. Lourenço C, Oliveira N, Ramos F, Ferreira I, Oliveira M. Aggressive angiomyxoma of the vagina: A case report. Rev Bras Ginecol Obstet. 2013;35(12):575-82. https://doi.org/10.1590/s0100-72032013001200008

17. Chauhan MB, Malhotra V, Dahiya P, Nanda S. Aggressive angiomyxoma of the vagina masquerading as genital prolapse. J Gynecol Surg. 2010;26(4: 277-9. https://doi.org/10.1089/gyn.2009.0044

18. Jiménez-Vieyra CR, Quintana-Romero V, AguileraMaldonado LV, Solís-Moreno TK. Angiomixoma agresivo de vagina: un tumor poco diagnosticado. Ginecol Obstet Mex. 2013;81(7):403-8.

19. Wang YF, Qian HL, Jin HM. Local recurrent vaginal aggressive angiomyxoma misdiagnosed as cellular angiomyofibroblastoma: A case report. Exp Ther Med. 2016;11(5):1893-5. https://doi.org/10.3892/ etm.2016.3097

20. Erol B, Pelit ES, Bektaş S, Şimşek A. Aggressive vaginal angiomyxoma mimicking a bladder mass. Urol J. 2014;11(3):1710-3. https://doi.org/10.22037/ uj.v11i3.2069

21. Shahid N, Ahluwalia A, Sahasrabudhe N, Davenport A. Aggressive angiomyxoma of the vagina: A rare differential diagnosis of a lateral vaginal wall cyst. J Obstet Gynaecol. 2005;25(6):622-3. https://doi. org/10.1080/01443610500243802

22. Stumpf AM, Stival SM, Merlini AB, Mansani FP, Silvestre JBCH, Mongruel IB, et al. Aggressive angiomyxoma of the vagina: A case report. J Bras Patol Med Lab. 2016;52(1):35-8. https://doi.org/10.5935/16762444.20160011

23. Seppings SC, Samir H, Abdelrahman I. An unusual vaginal lump. BMJ Case Rep. 2017; 2017:bcr2016218027. https://doi.org/10.1136/bcr-2016-218027

24. Cinel L, Taner D, Nabaei M, Dogan M. Aggressive angiomyxoma of the vagina: Report of a distinctive type gynecologic soft tissue neoplasm. Acta Ob- stet Gynecol Scan. 2000;79(3):232-3. https://doi. org/10.1080/j.1600-0412.2000.079003232.x

25. Larouche M, Hague CJ, Masoudi H, Geoffrion R. Aggressive angiomyxoma presenting as massive irreducible vaginal prolapse. Int Urogynecol J. 2016;27(12):1933-4. https://doi.org/10.1007/ s00192-016-3112-7

26. Amr SS, El-Mallah KO. Aggressive angiomyxoma of the vagina. Int J Gynecol Obstet. 1995;48(2):207-10. https://doi.org/10.1016/0020-7292(94)02266-2

27. Gonzaga LF, Freitas CM, Tavares JM. Aggressive vaginal angiomyxoma mimicking urethral tumor. Int Braz J Urol. 2005;31(5):475-6. https://doi.org/10.1590/ s1677-55382005000500011

28. Manandhar T, Sitaula S, Dixit B, Takur A. Angiomyxoma of the vagina mimicking as episiotomy scar endometriosis. A case report. European J Biomed Pharm Sci. 2019;(12):271-3.

29. Güngör T, Zengeroğlu S, Kaleli A, Kuzey G M. Aggressive angiomyxoma of the vulva and vagina. Eur J Obstet Gynecol Reprod Biol. 2004;112(1):114-6. https://doi.org/10.1016/s0301-2115(03)00270-7

30. Dahiya K, Jain S, Duhan N, Nanda S, Kundu P. Aggressive angiomyxoma of vulva and vagina: A series of three cases and review of literature. Arch Gynecol Obstet. 2010;283(5):1145-48. https://doi.org/10.1007/ s00404-010-1575-1

31. Herrera-Castro JC, González-Calderón G, MedinaFranco H. Angiomixoma agresivo: reporte de un caso y experiencia global con 168 casos. Ginecol Obstet Mex. 2017;85(11):755-62. https://doi.org/10.24245/ gom.v85i11.1504

32. Piura B, Shaco-Levy R. Pedunculated aggressive angiomyxoma arising from the vaginal suburethral area: Case report and review of literature. Eur J Gynaecol Oncol. 2005;26(5):568-71.

33. Zhu H, Wang S. Aggressive angiomyxoma of the vagina. Chin Med J (Engl). 2014;127(5):998.

34. Papachristou DJ, Batistatou A, Paraskevaidis E, Agnantis NJ. Aggressive angiomyxoma of the vagina: A case report and review of the literature. Eur J Gynaecol Oncol. 2004;25(4):519-21. PMID: 15285321. 


\section{CONTRIBUCIÓN DE LOS} AUTORES

Diana Meliza Hidalgo-Zambrano: elaboración del manuscrito, diseño metodológico, adquisición de la información, revisión del contenido intelectual, elaboración y adquisición del material grafico.
Daniel Felipe Reyes-Vega: elaboración del manuscrito, diseño metodológico, adquisición de la información, revisión del contenido intelectual, elaboración y adquisición del material gráfico.

Luis Guillermo Cano-Arias: elaboración del manuscrito, diseño metodológico, revisión del contenido intelectual y aprobación de la versión enviada a proceso editorial. 